\title{
DNA supercoiling and bacterial gene expression.
}

\section{Charles J Dorman}

Department of Microbiology,

Trinity College,

Dublin 2,

Ireland.

Tel +35318962013

Fax +35316799294

E-mail cjdorman@tcd.ie

Key words; DNA supercoiling, DNA topoisomerase, DNA gyrase, negative supercoiling, positive supercoiling, transcription, nucleoid-associated proteins, Fis, IHF, H-NS. 


\begin{abstract}
DNA in bacterial cells is maintained in a negatively supercoiled state. This contributes to the organization of the bacterial nucleoid and also influences the global gene expression pattern in the cell through modulatory effects on transcription. Supercoiling arises as a result of changes to the linking number of the relaxed double-stranded DNA molecule and is set and reset by the action of DNA topoisomerases. This process is subject to a multitude of influences that are usually summarized as environmental stress. Responsiveness of linking number change to stress offers the promise of a mechanism for the wholesale adjustment of the transcription programme of the cell as the bacterium experiences different environments. Recent data from DNA microarray experiments support this proposition. The emerging picture is one of DNA supercoiling acting at or near the apex of a regulatory hierarchy where it collaborates with nucleoid-associated proteins and transcription factors to determine the gene expression profile of the cell.
\end{abstract}




\section{Emerging opportunities in gene regulatory studies}

The application of genomics methodologies is revolutionizing analysis of bacterial gene regulation. Very large data sets are being produced in gene expression studies and a major challenge concerns the need to identify within these data patterns of regulation that will lead to useful mechanistic insights. One approach that is proving to be quite successful involves identifying regulators that have very wide effects on gene expression and then identifying all the genes that each controls collectively. By performing this operation for each pleiotropic regulator one can assemble a picture of the genetic complement of the bacterium where the genes are organized into sets. Overlaps between these sets provide important insights into the networking of the gene expression programme of the cell, at least under the conditions in which the measurements were made. It is important to emphasize that work on what one might term global regulatory mechanisms is not new. What is new is the powerful alliance between this type of investigation and the emerging technologies of transcriptomics, proteomics, metabolomics, interactomics etc. It is becoming apparent that earlier suggestions that DNA supercoiling would play a critical role in the global control of $\operatorname{transcription}^{1}$ were well founded. Currently we are at the stage of assembling what is proving to be an exciting and highly detailed picture of how the bacterial cell controls the expression of its genetic material.

\section{DNA supercoiling and nucleoid organization}

It is useful to begin a discussion of DNA supercoiling in bacteria by referring not to its effects on transcription but by considering its role in nucleoid packaging ${ }^{2}$. The 
chromosome of a bacterium such as Escherichia coli contains approximately 4.7 million bases and is about $1.5 \mathrm{~mm}$ in length. Packing this molecule into a capsule-shaped cell measuring 1 micron across by 2 microns long presents certain practical difficulties. These are solved by a combination of long-range and short-range structuring processes. It is now accepted that the chromosome is divided into a number of independent looped domains. The actual number has been difficult to determine but following recent estimates an emerging consensus indicates that it may be as high as 400 per chromosome $^{3-5}$. Thus looped domains may be quite small and some may accommodate just single operons. The nature of the boundaries between domains has been a matter for debate. No single factor has been identified that definitely constitutes a boundary element, either at the level of a cis-acting sequence in the chromosomal DNA or a transacting protein or RNA molecule. Nevertheless, there are many candidates, not least the nucleoid proteins in the case of trans-acting factors, of which more will be said below, and repetitive DNA sequences in the case of $c i s$-acting motifs $^{1-5}$. One feature of domain boundaries that has been established satisfactorily is the fact that they are ephemeral. Boundaries exist in abundance in the chromosomes of bacteria growing logarithmically but become rare as the bacteria enter stationary phase. This points to a highly dynamic nucleoid structure.

The looped domains are negatively supercoiled because they contain a deficit of DNA helical turns. The DNA responds by adopting a minimal energy conformation that is characterized by the writhing, and even branching, of the helical axis that one associates with plectonemically-wound negatively supercoiled DNA ${ }^{2}$. An early and attractive proposition that chromosomal domains are supercoiled to different levels has 
been examined in a number of studies. In this model, the genes in the different domains experience different levels of linking deficit in their DNA, possibility providing for different influences on expression. The model also raises the possibility that differentially supercoiled independent domains could act as a constraint on the locations in the chromosome where certain genes might be located and hence affect chromosome evolution. Experiments in which supercoiling-sensitive probes where placed at different locations on the chromosomes of E. coli $^{6}$ or Salmonella enterica serovar Typhimurium ${ }^{7}$ produced no data that supported the differentially-supercoiled domain thesis. Instead, any variations in gene expression detected by the probes could all be accounted for on the basis of gene copy number as a function of distance from the origin of chromosome replication, oriC.

The current model of the bacterial nucleoid incorporates looped domains constrained by boundaries that are transient and where the overall linking deficit is approximately equal in all domains. There may be roles for nucleoid-associated proteins in boundary formation. However, at least one report suggests that many major nucleoidassociated proteins can be eliminated from the cell without affecting the gross structure of the nucleoid ${ }^{8}$. It is also important to consider the possibility that some of these proteins, especially Fis, may have important structuring roles at the apices of loops where they might inhibit DNA slithering ${ }^{9,10}$.

\section{Supercoiling and DNA-based transactions}

The underwinding of the DNA in bacterial cells imparts free energy to the duplex that is available to do thermodynamic work ${ }^{11}$. The free energy of supercoiling varies as a 
function of the change in the linking number squared ${ }^{12}$. This relationship between the reduction in the linking number of the double-stranded DNA and free energy is such that even relatively modest reductions in linking number have the potential to influence the major transactions that take place in DNA. While these certainly include transcription they also encompass recombination (homologous, site-specific and transposition) and replication. Other DNA structural transitions, such as the conversion of B DNA to the Z form, the formation of triple-stranded H-DNA and cruciform extrusion can also be driven by reductions in linking number ${ }^{12}$.

Changes to the linking number usually require breakage and reunion of the DNA strands, reactions that are catalyzed by topoisomerases ${ }^{13}$. Bacteria possess a unique enzyme called DNA gyrase that catalyses this reaction ${ }^{14}$. In E.coli, gyrase is a tetrameric protein with an $\mathrm{A}_{2} \mathrm{~B}_{2}$ structure. The $g y r A$ and $g y r B$ genes encode the $A$ and $B$ subunits, respectively. Knockout mutations in these genes are lethal, indicating that gyrase is an essential enzyme. It is a type II topoisomerase and this means that it alters the linking number of its DNA substrate in steps of two. The underwinding of DNA that accompanies linking number reduction requires energy and gyrase consumes ATP in this process. This has the physiologically important effect of linking gyrase activity directly to the metabolic state of the cell ${ }^{1}$. The activity of gyrase is opposed by DNA topoisomerase I, a type I enzyme that alters the linking number in steps of one ${ }^{14}$. Like gyrase, topoisomerase I requires $\mathrm{Mg}^{2+}$, but unlike gyrase it does not require ATP. Instead, topoisomerase I gets the energy to drive its DNA relaxation reaction from the DNA substrate itself. It cuts one DNA strand and allows it to swivel around the intact strand, relaxing the molecule in the process. For this reason, topoisomerase I is sometimes 
referred to as a swivelase. This protein is monomeric and is encoded by the topA gene. Mutations in topA are not lethal but are debilitating for the cell. Part of the reason that knockout mutations in topA can be tolerated concerns the existence of other topoisomerases in the cells that can relax DNA, including topoisomerase $\mathrm{IV}^{15,16}$. This type II topoisomerase is structurally closely related to gyrase but lacks the ability to underwind the DNA substrate ${ }^{17}$. Early genetic studies showed that mutations in the topA gene could be compensated by second site mutations that down-regulated the activity of gyrase or amplified the number of copies of the genes on the chromosome ( $\operatorname{par} C$ and parE) that code for topoisomerase $\mathrm{IV}^{15,16}$. It was also found that protecting the mutant bacteria from osmotic stress or anaerobic growth conditions could ameliorate the severity of the physiological effects associated with a topA mutation ${ }^{18}$. These latter observations pointed to an important link between the environment and DNA supercoiling. Molecular analysis revealed that the promoters of the $g y r A$ and $g y r B$ genes are activated by DNA relaxation, whereas the promoter of the topA gene is stimulated by DNA underwinding. These observations led to a homeostatic model of DNA supercoiling, in which the activity of each major topoisomerase stimulates the expression of its countervailing partner. The outcome is a 'balanced' level of DNA supercoiling that is presumed to be optimal for cellular survival $^{12,20}$.

\section{Transcription and DNA supercoiling}

DNA supercoiling has the potential to influence transcription at a number of levels ${ }^{21}$. The most obvious, perhaps, concerns the isomerization step in transcription initiation where the promoter-RNA polymerase closed complex is opened. The ability of negative 
supercoiling to assist double stranded DNA to open to form a single-stranded region follows easily from the intuitive notion of underwound DNA. Energy is needed to break the hydrogen bonds and this can be supplied by underwinding the DNA. However, before the process reaches the point of open complex formation, RNA polymerase must be recruited to the promoter and supercoiling can influence this step in different ways. One of these concerns promoters in which the canonical -10 and -35 hexameric elements recognized by the sigma factor of RNA polymerase are separated by a spacer of nonstandard length. Most spacers in promoters that are read by the Sigma-70 housekeeping sigma factor are $17 \mathrm{bp}$ in length. Adding or subtracting bases alters the relative distribution of the -10 and -35 along the face of the DNA helix. This rotational distortion can be corrected by altering the twist of the DNA. This is possible because the consequences of linking number reduction are typically partitioned between the twist and writhe of the DNA according to the formula $\Delta \mathrm{Lk}=\Delta \mathrm{Tw}+\Delta \mathrm{Wr}^{12,21}$.

The recruitment of RNA polymerase to a promoter can also depend on the presence of a transcription factor and the binding of this regulatory protein can be influenced by the topology of the DNA at its binding site ${ }^{22}$. Similarly, DNA supercoiling can influence the interaction of the transcription factor with RNA polymerase profoundly. For example, the closed transcription initiation complexes formed by Sigma-54dependent promoters are normally completely silent until a transcription factor that is bound to a far upstream sequence (called an enhancer) is brought into intimate physical contact with RNA polymerase ${ }^{22}$. The delivery of the upstream-located transcription factor is facilitated by the introduction of writhing turns in the intervening DNA through linking number reduction. Promoters that are subject to stringent control in response to the 
alarmones ppGpp and pppGpp possess $\mathrm{G}+\mathrm{C}$-rich discriminator regions immediately downstream of their -10 motifs that are an impediment to transcription ${ }^{21}$. These promoters drive the expression, inter alia, of genes involved in the expression of the translation machinery of the cell. This machinery must be down regulated when the bacterium is starved and the stringent response is a key element in the achievement of this. The G+C-rich region is difficult to melt because it is more tightly hydrogen bonded than the typically A+T-rich sequences found at most bacterial promoters. The discriminator function relies on the need for additional energy, in the form of underwound DNA, to overcome the activation barrier that is imposed by the $\mathrm{G}+\mathrm{C}$-rich sequence $^{21}$. This additional energy, channeled through DNA gyrase activity, is more likely to be available in bacteria with a high metabolic flux and a correspondingly high $[\mathrm{ATP}] /[\mathrm{ADP}]$ ratio than in starved organisms $\mathrm{s}^{12,21}$.

Echoes of this discriminatory function can be detected in the case of promoters that are read by the so-called stress- and stationary-phase-specific sigma factor, Sigma38, or RpoS. These promoters are often difficult to distinguish by sequence analysis from those that are read by Sigma-70 although they do have distinct features. One of the characteristics of an RNA polymerase that has been reprogrammed by Sigma-38 is an ability to utilize promoters in relaxed DNA much more efficiently than is the case for polymerases containing Sigma- $70^{23}$. Significantly, relaxation is a feature of DNA in bacteria that are in the stationary phase of growth, precisely the period when Sigma-38dependent transcription is required. Although this discussion focuses on transcription initiation, it should be remembered that supercoiling of the DNA template also has the potential to influence later stages in the process, such as elongation and termination. 
It is important to emphasize that while DNA supercoiling can influence transcription, transcription also affects supercoiling ${ }^{24}$. This arises because of the physical conditions under which the process occurs in the cell. As RNA polymerase tracks along DNA, the moving complex causes the DNA ahead to become overwound and that behind to be underwound. This is equivalent to the creation of a positively supercoiled domain ahead and a negatively supercoiled domain behind the moving complex. It follows that the transcription complex will quickly reach a point where further progress becomes physically impossible unless the resulting torsional strain is relieved by (1) the rotation of the complex around the DNA molecule (2) the rotation of the DNA template or (3) the intervention of agents capable of resetting the topology of the DNA. It is believed that rotation of the transcription complex is unlikely to be easy because of the viscous drag experienced in the crowded environment of the cytoplasm. This effect is exacerbated by the development of the growing RNA transcript, and is further exacerbated by the addition of ribosomes and nascent polypeptides in the case of mRNA. These combine to exert an effect analogous to that of an anchor on the rotation of the transcription complex ${ }^{24}$. Free rotation of the DNA template is likely to be inhibited by anchorage at domain boundaries. It is believed that in practice, the topological problems associated with the movement of the transcription complex are solved by the intervention of topoisomerases. This is supported by experimental evidence from a number of model systems ${ }^{1}$. This is also the case for other DNA-based tracking processes, such as DNA replication. DNA gyrase can relax the positive supercoils that form ahead of the moving complex while DNA topoisomerase I relaxes the negative supercoils that form behind. However, it is necessary for these topoisomerases to be available for this to occur, so 
circumstances that interfere with the normal expression of these enzymes or their activities may have unforeseen consequences at a local level of some promoters.

A key corollary of the discovery that transcription affects DNA supercoiling is that promoters may influence one another at a local level. Back-to-back promoters can pump negative supercoiling towards each other, with mutual influences on transcription performance. Convergent promoters have the potential to drive each other into a positively supercoiled state, again with potential effects on performance. Promoters arranged in tandem can influence upstream and downstream neighbours for the same reasons. There is a considerable body of experimental data that supports these models of promoter-promoter coupling. In one case a series of promoters along the chromosome of Salmonella in the neighbourhood of the leuABCD operon has been shown to participate in a relay-based mutual activation mechanism ${ }^{25}$. The phenomenon of promoter coupling has important implications for our understanding of how promoter activity can be influenced at a very local level by the effects of transcriptionally generated changes in DNA supercoiling. It also has implications for our views of the possible permissible arrangements of genes and their promoters along the chromosome or other replicon.

\section{Environmental influences on DNA supercoiling}

Potentially, every promoter in the cell can be affected by changes in DNA supercoiling. The homeostatic model indicates that bacteria strive to maintain the superhelical density of DNA close to an optimal value. Superhelical density, $\sigma$, is related to the linking number change of DNA as follows: $\sigma=\Delta \mathrm{Lk} / \mathrm{Lk}^{0}$, where $\Delta \mathrm{Lk}$ is the change in linking number and $\mathrm{Lk}^{0}$ is the linking number of the same DNA molecule in its fully relaxed 
state $^{11}$. The value of $\sigma$ has been estimated as -0.025 for DNA in bacteria such as $E$. coli. To be useful in a gene regulatory role, supercoiling levels would have to be variable. Several lines of evidence support the proposition that it does vary. Importantly, the linking number of DNA has been shown to change when bacteria move from low to high osmolarity growth media and from aerobic to anaerobic growth conditions ${ }^{26,27}$. It is also affected by growth phase, temperature and $\mathrm{pH}^{27-29}$. In each case, the resulting change in supercoiling has been shown to alter the transcription of large groups of genes ${ }^{30}$.

This leads naturally to a hierarchical view of gene regulation in bacteria ${ }^{1,12,21}$. Here, supercoiling occupies a position at the apex of the pyramid where it can affect a very wide range of genes. The members of the family of nucleoid-associated proteins exert further wide-ranging effects on gene expression. Transcription factors such as the cAMP-receptor protein, Crp and the leucine-responsive regulatory protein, Lrp come next and finally the so-called conventional transcription factors, each influencing just a handful of genes. Promoter-promoter coupling mechanisms probably contribute to finetuning of the gene expression programme. Evidence supporting the wide-ranging effects of DNA supercoiling on gene expression come from many studies of individual genes and from transcriptomic analyses where the effects on gene expression patterns of mutations in genes coding for topoisomerases and treatments with antibiotics that inhibit topoisomerase function have been assessed ${ }^{31,32}$. Transcriptomic analysis has also confirmed the link between the response to osmotic shock and DNA supercoiling sensitivity of genes involved in that process $^{30}$. 


\section{Nucleoid-associated proteins}

There is a discrepancy in measurements of the superhelical density of DNA within the bacterial cell $(-0.025)$ and the same DNA purified from the cell $(-0.05)^{11}$. This is accounted for if approximately half of the supercoils in DNA are constrained while it is inside the cell. Consequently, it is believed that half of the bulk DNA exists in complexes with protein and half is plectonemically wrapped in bacteria growing logarithmically. The DNA isolation procedure removes the proteins and reveals the full extent to which the DNA is supercoiled. There is no evidence that some DNA is permanently associated with protein in the cell and some permanently protein-free. On the contrary, all indications are that the situation is highly dynamic. There are many proteins involved in forming these nucleoprotein complexes, of which RNA polymerase is clearly an important example. Others include the many members of the nucleoid-associated protein family. This group includes the Factor for Inversion Stimulation (Fis), a protein that influences DNA topology in bacteria at a number of levels ${ }^{10,33}$.

\section{(a) The Fis protein}

Fis is a homodimeric DNA binding protein that binds to a rather degenerate consensus sequence. Originally identified as an important co-factor in site-specific recombination reactions in bacteria and bacteriophage, it is now recognized as having wide-ranging effects on transcription ${ }^{34,35}$. The Fis protein is expressed by fis, a gene whose promoter is activated by negative supercoiling. Fis has been identified as a transcriptional repressor of the $g y r A$ and $g y r B$ genes and, under certain circumstances it is an activator of top $A$ transcription. This gives Fis a direct role in modulating the supply of topoisomerases in 
the cell. In addition, it can modulate their activities. This is because the Fis protein has a preference for binding to DNA molecules of intermediate superhelical density and protecting them from further linking number changes. For this reason, it has been described as a topological buffer ${ }^{36,37}$. Naturally, the ability of the Fis protein to influence events in the cell is contingent on its being present. The protein shows a striking growthphase-dependent expression pattern that is matched by fis mRNA, where there is a spike of expression in early logarithmic growth as the cells exist lag phase. Fis plays a key role here in the up-regulation of genes involved in the production of the translation machinery of the cell. These same genes are usually exquisitely sensitive to changes in DNA supercoiling. The mechanism by which Fis acts at these promoters seems to involve a combination of those properties that one associates with conventional transcription regulators (e.g. direct contact with RNA polymerase) and its role as a topological buffer where it organizes a micro-domain of negatively supercoiled DNA immediately upstream of the promoter, preserving a structural conformation that is optimal for promoter function. Studies of Fis at the promoters of stringently regulated stable RNA genes have revealed a feature that recurs at other Fis-regulated promoters: an antagonistic relationship with the nucleoid-associated protein H-NS.

\section{(b) The H-NS protein}

The H-NS protein is an abundant DNA binding protein that is present in the cell approximately at a constant level throughout growth ${ }^{38}$. It can constrain DNA supercoils both in vivo and in vitro and is a highly pleiotropic regulator of transcription. In every case where the contribution of H-NS has been shown to be direct, it acts as a repressor. 
All the indications are that H-NS exists in the cell to down-regulate transcription. If this is so, how is its negative influence counteracted? Part of the answer comes from attempts to identify the features in DNA to which this protein binds. It has proved to be extremely difficult to identify a consensus DNA sequence for H-NS binding. Instead, the protein seems to have a preference for binding to particular structures, mainly regions of intrinsic planar curvature. Frequently, such structural motifs are specified by A+T-rich DNA sequences and are found at many bacterial promoters. Modulation of the H-NS-DNA interaction is likely to involve changes to the nucleic acid rather than to the protein. There is no strong evidence that H-NS (or Fis) binds a ligand, is covalently modified or undergoes proteolytic cleavage. However, the maintenance of curvature in DNA is contingent on the chemical and physical environment. For example, curvature is ablated by increases in temperature ${ }^{39}$. It is interesting to note that many H-NS-repressed genes are activated by rising temperature. Another mechanism for antagonizing the repressive activity of H-NS involves remodelling of the DNA by the binding of another protein. This could be a conventional transcription factor (which also contacts RNA polymerase) or a protein that simply removes H-NS (an anti-repressor). The Fis protein is a good example of an anti-H-NS factor, and there is an interesting correspondence between the memberships of the H-NS ${ }^{40,41}$ and Fis ${ }^{34}$ regulons in Salmonella.

\section{(c) The IHF protein}

The integration host factor (IHF) is a nucleoid-associated protein that acts locally at many genes and has been shown to cooperate intimately with negative supercoiling to influence promoter function. Originally identified as an essential component of the bacteriophage 
lambda integration and excision process, IHF is now established as a major influence on the global gene expression pattern of E. coli, Salmonella, and other bacteria ${ }^{42,43}$.

The IHF protein has been implicated in the redistribution of DNA twist at a local level on the chromosome. This phenomenon involves DNA elements subject to stressinduced duplex destabilization (SIDD) ${ }^{20,44}$. As the name suggests, these elements are particularly prone to the formation of single stranded regions in response to the stress associated with DNA underwinding. In the case of the $i l v P G$ promoter of E. coli, binding of the IHF protein at the SIDD sequence and the associated bending of the DNA resulted in transmission of the tendency towards DNA duplex unwinding from the SIDD site to the Pribnow box of the promoter. In principle, this DNA twist transmission mechanism need not be confined to any one protein; any DNA binding protein capable of bending DNA should suffice. In keeping with this proposition, the Fis protein has also been shown by experiment to be able to activate a promoter at a stable RNA gene using DNA unwinding energy from a SIDD element ${ }^{45}$.

\section{DNA supercoiling and bacterial virulence}

A recurring theme in this article has been the responsiveness of DNA topology in bacterial cells to environmental stress. It was proposed some time ago that this was likely to be exploited by bacterial pathogens as a means of regulating virulence gene expression during infection ${ }^{46}$. There is now a wealth of evidence to show that this is the case in many pathogenic species. The Gram-negative pathogens Salmonella and Shigella have been examined in particular detail and it is very interesting to see the major components of the nucleoid-structuring apparatus being implicated in aspects of the control networks 
governing the repertoires of virulence genes possessed by these bacteria. Both are facultative intracellular pathogens that rely on type-three secretion systems to interact successfully with their hosts. Genes with an abnormally high A+T content that are thought to have been acquired horizontally en bloc from unknown sources during the evolution of the pathogens encode these complex structures. These genes are found within so-called pathogenicity islands on the chromosome in the case of Salmonella and on a large plasmid in the case of Shigella ${ }^{47,48}$. In both cases temperature, osmolarity and $\mathrm{pH}$ play important roles in modulating transcription. It is thought that this helps to inform the microbe about its location within the host so that the type-three secretion systems are only deployed and used when the bacterium is in the gut.

Although most molecular detail is available for the Shigella flexneri system, it is clear that the virulence gene promoters in the pathogenicity islands of these bacteria are repressed by $\mathrm{H}-\mathrm{NS}^{47}$. The strong affinity of H-NS for these horizontally acquired genes has prompted speculation that this may be a mechanism for selectively down-regulating genes of this type until the bacterium has had an opportunity to embed them appropriately in its existing gene regulatory circuits ${ }^{40,41}$. The H-NS repressor in S. flexneri is opposed by transcription factors that appear in the cell in response to the correct profile of environmental signals. It is also opposed by the Fis protein and counteracted by a DNA structural transition that removes intrinsic DNA curvature ${ }^{38}$. The regulatory and structural genes all possess promoters that are response to DNA linking number change, providing a classic example of virulence gene regulation by nucleoid-structuring factors, including negative supercoiling of the DNA ${ }^{47}$. 


\section{Perspective}

Much work remains to be done in the field of gene regulation by DNA supercoiling. Its contributions to the control of virulence gene expression in pathogens hint at possible applied benefits from greater knowledge of this topic. However, the available information is still very incomplete. For example, it has been shown recently that linking number changes in DNA collaborate with the Fis protein to modulate virulence gene expression in Salmonella while the bacteria are inside mammalian cells ${ }^{49}$. Studies of bacterial transcription in the intracellular arena are in their infancy and many more data are needed to build reliable models of the key adaptation events during the adjustment of the microbial gene expression programme to life inside host cells. More kinetic data are also needed so that the gene expression pattern may be considered in four dimensions to provide useful knowledge on the spatiotemporal programme of gene regulation in all environments relevant to the lives of the most important microbes. This ambition moves the field firmly into the realm of systems biology where one can be confident that studies of DNA supercoiling-mediated effects on gene expression will make significant contributions to a full understanding of microbial life.

\section{Acknowledgements}

The author acknowledges financial support from Science Foundation Ireland and the Wellcome Trust. 


\section{References}

1. Dorman, C.J. (1994) Genetics of Bacterial Virulence. Blackwell Science, Oxford.

2. Trun, N.J. \& Marko, J.F. (1998) Architecture of the bacterial chromosome. ASM Press 64(5), 276-283.

3. Stein, R.A., Deng, S. \& Higgins, N.P. (2005) Measuring chromosome dynamics on different time scales using resolvases with varying half-lives. Mol Microbiol 56, 10491061.

4. Deng, S., Stein, R.A., \& Higgins, N.P. (2005) Organization of supercoil domains and their reorganization by transcription. Mol Microbiol 57, 1511-1521.

5. Postow, L., Hardy, C.D., Arsuaga, J. \& Cozzarelli, N.R. (2004) Topological domain structure of the Escherichia coli chromosome. Genes Dev 18, 1766-1779.

6. Miller, W.G. \& Simons, R.W. (1993) Chromosomal supercoiling in Escherichia coli. Mol Microbiol 10, 675-684.

7. Pavitt, G.D. \& Higgins, C.F. (1993) Chromosomal domains of supercoiling in Salmonella typhimurium. Mol Microbiol 10, 685-696.

8. Zimmerman, S.B. (2006) Cooperative transitions of isolated Escherichia coli nucleoids: implications for the nucleoid of a cellular phase. J Struct Biol 153, 160-175. 9. Schneider, R., Lurz, R., Lüder, R., Tolksdorf, C., Travers, A. \& Muskhelishvili, G. (2001) An architectural role for the Escherichia coli chromatin protein FIS in organizing DNA. Nucleic Acids Res 2001, 29, 5107-5114.

10. Dorman, C.J. \& Deighan, P. (2003) Regulation of gene expression by histone-like proteins in bacteria. Curr Opin Genet Dev 13, 179-184.

11. Sinden, R.R. (1994) DNA Structure and function. Academic Press, San Diego. 
12. Dorman, C.J. (2002) DNA topology and regulation of bacterial gene expression. SGM Symp 61, 39-56.

13. Giaever, G.N., Snyder, L. \& Wang, J.C. (1988) DNA supercoiling in vivo. Biophys Chem 29, 7-15.

14. Drlica, K. (1992) Control of bacterial DNA supercoiling. Mol Microbiol 6, 425-433. 15. DiNardo, S., Voelkel, K.A., Sternglanz, R., Reynolds, A.E. \& Wright, A. (1983) Escherichia coli DNA topoisomerase I mutants have compensatory mutations at or near DNA gyrase genes. Cold Spring Harbor Symp Quant Biol 47, 779-784.

16. Free, A. \& Dorman, C.J. 1994. Escherichia coli tyrT gene transcription is sensitive to DNA supercoiling in its native chromosomal context: effect of DNA topoisomerase IV over-expression on tyrT promoter function. Mol Microbiol 14, 151-161.

17. Drlica, K. \& Zhao, X. (1997) DNA gyrase, topoisomerase IV, and the 4-quinolones. Microbiol Mol Biol Rev 61, 377-392.

18. Dorman, C.J., Lynch, A.S., Ní Bhriain, N. \& Higgins, C.F. (1989) DNA supercoiling in Escherichia coli: topA mutations can be suppressed by DNA amplifications involving the tolC locus. Mol Microbiol 3: 531-540.

19. Kato, J.-L., Nishimura, Y., Imamura R., Niki, H., Hiraga, S. \& Suzuki, H. (1990) New topoisomerase essential for chromosome segregation in E. coli. Cell 63, 393-404. 20. Hatfield, G.W. \& Benham, C.J. (2002) DNA topology-mediated control of global gene expression in Escherichia coli. Annu Rev Genet 36, 175-203.

21. Travers, A. \& Muskhelishvili, G. (2005) DNA supercoiling - a global transcriptional regulator for enterobacterial growth? Nat Rev Microbiol 3, 157-169. 
22. Wagner, R. (2000) Transcription Regulation in Prokaryotes. Oxford University Press.

23. Bordes, P., Conter, A., Morales, V., Bouvier, J., Kolb, A. \& Gutierrez C. (2003) DNA supercoiling contributes to disconnect sigmaS accumulation from sigmaS-dependent transcription in Escherichia coli. Mol Microbiol 48, 561-571.

24. Liu, L.F. \& Wang, J.C. (1987) Supercoiling of the DNA template during transcription. Proc Natl Acad Sci USA84, 7024-7027.

25. Wu, H.Y., Tan, J. \& Fang, M. (1995) Long-range interaction between two promoters: activation of the leu-500 promoter by a distant upstream promoter. Cell $\mathbf{8 2}, 445-451$. 26. Higgins, C.F., Dorman, C.J., Stirling, D.A., Waddell, L., Booth, I.R., May, G. \& Bremer, E. (1988) A physiological role for DNA supercoiling in the osmotic regulation of gene expression in S. typhimurium and E. coli. Cell 52, 569-584.

27. Dorman, C.J., Barr, G.C., Ní Bhriain, N. \& Higgins, C.F. (1988) DNA supercoiling and the anaerobic and growth-phase regulation of tonB gene expression. $J$ Bacteriol 170, 2816-2826.

28. Dorman, C.J., Ní Bhriain, N. \& Higgins, C.F. (1990) DNA supercoiling and the environmental regulation of virulence gene expression in Shigella flexneri. Nature 344, 789-792.

29. Karem, K. \& Foster, J.W. (1993) The influence of DNA topology on the environmental regulation of a $\mathrm{pH}-$ regulated locus in Salmonella typhimurium. Mol Microbiol 10, 75-86. 
30. Cheung, K.J., Badarinarayana, V., Selinger, D.W., Janse, D. \& Church, G.M. (2003) A microarray-based antibiotic screen identifies a regulatory role for supercoiling in the osmotic stress response of Escherichia coli. Genome Res 13, 206-215.

31. Peter, B.J., Arsuaga, J., Breier, A.M., Khodursky, A.B., Brown, P.O. \& Cozzarelli, N.R. (2004) Genome transcriptional response to loss of chromosomal supercoiling in Escherichia coli. Genome Biol 5:R87.

32. Blot, N., Mavathur, R., Geertz, M., Travers, A., \& Muskhelishvili, G. (2006) Homeostatic regulation of supercoiling sensitivity coordinates transcription of the bacterial genome. EMBO Rep 7, 710-715.

33. Travers, A. \& Muskhelishvili, G. (2005) Bacterial chromatin. Curr Opin Genet Dev 15, 507-514.

34. Kelly, A., Goldberg, M.D., Carroll, R.K., Danino, V., Hinton, J.C.D. \& Dorman, C.J. (2004) A global role for Fis in the transcriptional control of metabolic and type III secretion genes of Salmonella enterica serovar Typhimurium. Microbiol 150, 2037-2053. 35. Travers, A., Schneider, R. \& Muskhelishvili, G. (2001) DNA supercoiling and transcription in Escherichia coli: The FIS connection. Biochimie 83, 213-217.

36. Rochman, M., Blot, N., Dyachenko, M., Glaser, G., Travers, A. \& Muskhelishvili, G. (2004) Buffering of stable RNA promoter activity against DNA relaxation requires a far upstream sequence. Mol Microbiol 53, 143-152.

37. Muskhelishvili, G. \& Travers, A. (2003) Transcription factor as a topological homeostat. Front Biosci 8, d279-285.

38. Dorman, C.J. (2004) H-NS: a universal regulator for a dynamic genome. Nature Rev Microbiol 2, 391-400. 
39. Prosseda, G., Falconi, M., Giangrossi, M., Gualerzi, C.O., Micheli, G. \& Colonna, B. (2004) The virF promoter in Shigella: more than just a curved DNA stretch. Mol Microbiol 51, 523-537.

40. Navarre, W.W., Porwollik, S., Wang, Y., McClelland, M., Rosen, H., Libby, S.J. \& Fang, F.C. (2006) Selective silencing of foreign DNA with low GC content by the H-NS protein in Salmonella. Science 313, 236-238.

41. Lucchini, S., Rowley, G., Goldberg, M.D., Hurd, D., Harrison, M. \& Hinton J.C.D. (2006) H-NS mediates the silencing of laterally acquired genes in bacteria.

PLoS Pathog Aug 18, 2(8).

42. Arfin, S.M., Long, A.D., Ito, E.T., Tolleri, L., Riehle, M.M., Paegle, E.S. \& Hatfield, G.W. (2000) Global gene expression profiling in Escherichia coli K12. The effects of integration host factor. J Biol Chem 275, 29672-29684.

43. Mangan, M.W., Lucchini, S., Danino, V., Ó Cróinín, T., Hinton, J.C.D. \& Dorman, C.J. (2006) The integration host factor (IHF) integrates stationary phase and virulence gene expression in Salmonella enterica serovar Typhimurium. Mol Microbiol 59, 18311847.

44. Parekh, B.S., Sheridan, S.D. \& Hatfield, G.W. (1996) Effects of integration host factor and DNA supercoiling on transcription from the ilvPG promoter of Escherichia coli. J Biol Chem 271, 20258-20264.

45. Opel, M.L., Aeling, K.A., Holmes, W.M., Johnson, R.C., Benham, C.J. \& Hatfield G.W. (2004) Activation of transcription initiation from a stable RNA promoter by a Fis protein-mediated DNA structural transmission mechanism. Mol Microbiol 53, 665-674. 
46. Dorman, C.J. (1991) DNA supercoiling and the environmental regulation of virulence gene expression in bacterial pathogens. Infect Immun 59, 745-749.

47. Dorman CJ. 2004. Virulence gene regulation in Shigella. In Escherichia coli and Salmonella: Cellular and Molecular Biology. (eds. R. Curtiss III, J.L. Ingraham, J.B. Kaper, S. Maloy, F.C. Neidhardt, M.M. Riley, C.J. Squires, B.L. Wanner, A. Bock) Third edition, EcoSal online. Posted 29th December, 2004 at http://www.ecosal.org. American Society for Microbiology, Washington, D.C.

48. Rhen, M. \& Dorman, C.J. 2005. Hierarchical gene regulators adapt Salmonella enterica to its host milieus. Int J Med Microbiol 295, 487-502.

49. Ó Cróinín, T., Carroll, R.K., Kelly, A. \& Dorman, C.J. (2006) Roles for DNA supercoiling and the FIS protein in modulating expression of virulence genes during intracellular growth of Salmonella enterica serovar Typhimurium. Mol Microbiol 62: 869-882. 